OPEN ACCESS

Edited by:

Stefan N. Constantinescu,

Université catholique

de Louvain, Belgium

Reviewed by:

Sandra Pellegrini,

Institut Pasteur (INSERM), France

Iris Behrmann,

University of Luxembourg,

Luxembourg

*Correspondence:

Patrick J. Lupardus

lupardus.patrick@gene.com

Specialty section: This article was submitted to Molecular and Structural Endocrinology,

a section of the journal

Frontiers in Endocrinology

Received: 19 January 2017

Accepted: 27 March 2017

Published: 18 April 2017

Citation:

Ferrao $R$ and Lupardus PJ (2017)

The Janus Kinase (JAK) FERM and SH2 Domains: Bringing Specificity

to JAK-Receptor Interactions.

Front. Endocrinol. 8:71.

doi: 10.3389/fendo.2017.00071

\section{The Janus Kinase (JAK) FERM and SH2 Domains: Bringing Specificity to JAK-Receptor Interactions}

\author{
Ryan Ferrao and Patrick J. Lupardus* \\ Department of Structural Biology, Genentech Inc., South San Francisco, CA, USA
}

The Janus kinases (JAKs) are non-receptor tyrosine kinases essential for signaling in response to cytokines and interferons and thereby control many essential functions in growth, development, and immune regulation. JAKs are unique among tyrosine kinases for their constitutive yet non-covalent association with class I and II cytokine receptors, which upon cytokine binding bring together two JAKs to create an active signaling complex. JAK association with cytokine receptors is facilitated by N-terminal FERM and $\mathrm{SH} 2$ domains, both of which are classical mediators of peptide interactions. Together, the JAK FERM and SH2 domains mediate a bipartite interaction with two distinct receptor peptide motifs, the proline-rich "Box1" and hydrophobic "Box2," which are present in the intracellular domain of cytokine receptors. While the general sidechain chemistry of Box1 and Box2 peptides is conserved between receptors, they share very weak primary sequence homology, making it impossible to posit why certain JAKs preferentially interact with and signal through specific subsets of cytokine receptors. Here, we review the structure and function of the JAK FERM and SH2 domains in light of several recent studies that reveal their atomic structure and elucidate interaction mechanisms with both the Box1 and Box2 receptor motifs. These crystal structures demonstrate how evolution has repurposed the JAK FERM and $\mathrm{SH} 2$ domains into a receptor-binding module that facilitates interactions with multiple receptors possessing diverse primary sequences.

Keywords: JAK1, JAK2, JAK3, TYK2, Interferon, FERM, SH2, Janus kinase

\section{INTRODUCTION}

Cytokines are a large family of secreted proteins with wide-ranging effects on cell growth, hematopoiesis, immunity, and inflammation $(1,2)$. Cytokines induce signaling by binding a specific set of cognate transmembrane receptors, a process that is thought to be initiated either by de novo dimerization of these receptors or by a conformational rearrangement of existing inactive receptor dimers $(3,4)$. Assembly of a functional cytokine-receptor signaling complex results in the activation and trans-phosphorylation of Janus kinases (JAKs), a family of cytoplasmic multi-domain tyrosine kinases (5). The four members of the JAK family, JAK1, JAK2, JAK3, and TYK2, are constitutively associated with the intracellular domains of cytokine receptors. Activated JAKs phosphorylate tyrosine residues within the cytokine receptor intracellular domains, which serve as binding sites for the STAT (signal transducer and activator of transcription) transcription factors (6). Recruitment of STATs to the receptor-JAK complex facilitates STAT phosphorylation by active JAKs, leading to 
STAT dimerization and translocation to the nucleus, where they promote transcription of cytokine-responsive genes.

Cytokine receptors can be divided into the class I and class II subfamilies based on structural similarities in both their ligands and their receptor sequences (7). The class I cytokine receptor family, which contains a conserved extracellular WSXWS motif within the second FNIII domain of the cytokine-binding homology region $(8,9)$, is made up of four subfamilies. Three of the subfamilies, the common gamma $\left(\gamma_{c}\right)$ family, the common beta $\left(\beta_{c}\right)$ family, and the gp130 family, are defined by their use of a shared (or common) signal transducing chain for the formation of homo- and hetero-oligomeric signaling complexes (10). An additional subfamily of class I cytokine receptors, which includes the growth hormone $(\mathrm{GH})$ and erythropoietin (EPO) receptors, signal as homodimeric pairs $(8,11)$. The class II cytokine receptor family contains receptors for the type I, type II, and type III interferons as well as the IL-10 cytokine family (12). Each cytokine receptor family interacts with and signals through a distinct subset of JAKs. GH and EPO receptors signal solely through JAK2, the $\beta_{c}$ family through JAK2, the $\gamma_{c}$ family through JAK1 and JAK3, and the gp130 and class II cytokine families through JAK1, JAK2, and/or TYK2 (7).

The intracellular domains of cytokine receptors are largely unstructured (13) and contain anywhere from several dozen to several hundred amino acids. Signaling through cytokine receptors was initially found to require the membrane proximal region of the intracellular domain (14), which was subsequently further defined as two distinct conserved motifs: a proline-rich segment termed "Box1" and a hydrophobic segment approximately 20-40 residues downstream called "Box2" $(15,16)$. The JAK kinases were identified in the early 1990s (17-20) and were quickly found to be essential for mediating signaling downstream of cytokine receptors (21-23). In addition to their C-terminal kinase domain, JAKs also contain a pseudokinase domain (psKD), an SH2 domain, and a FERM domain. Individual JAK family members were found to physically associate with various cytokine receptors (24-27), and the JAK FERM and SH2 domains were identified as essential for mediating the JAK-receptor interaction (28-34). More recently, the structure of the pseudokinase and kinase domains has been solved, elucidating the structural basis for regulation of kinase activity by the pKD (35-38).

Due to the essential role ofJAK-receptor interactions in cytokine signaling, naturally occurring mutations in both cytokine receptors and JAKs lead to immunodeficiency and myeloproliferative disorders (39). An autosomal severe combined immunodeficiency (SCID)-associated mutation, Y100C, is located in the hydrophobic core of the JAK3 FERM domain. This mutation disrupts the interaction with the $\gamma_{c}$ receptor chain and leads to constitutive JAK3 phosphorylation, likely due to aggregation-induced transphosphorylation of the destabilized JAK3 (40). Additionally, nonsense mutations that result in a truncated $\gamma_{c}$ lead to X-linked SCID, and the L271Q mutation in the Box 1 of $\gamma_{c}$ causes a more moderate form of X-linked combined immunodeficiency (41, 42). Despite a substantial amount of research performed on understanding the essential components of both the receptor and kinase, the molecular underpinnings of the JAK-receptor interaction have remained elusive. This review will focus on the recent advances in our understanding the molecular structure of JAK-receptor complexes.

\section{STRUCTURE OF THE JAK FAMILY FERM- SH2 RECEPTOR INTERACTION MODULE}

An atomic-level structural model of the JAK FERM and SH2 domains and the mechanism of JAK interaction with receptors remained elusive until recently, largely due to the intractability of JAK protein crystallization due to poor expression and solubility (43). Copurification with a stabilizing receptor enabled an initial X-ray crystal structure of the human TYK2 FERM and SH2 domains in 2014 (44) followed by crystal structures of the human JAK1 $(45,46)$ and JAK2 (47) FERM-SH2 fragments in 2016. These structures surprisingly revealed that the JAK FERM and SH2 domains are tightly associated to form a single receptor-binding module (Figures 1A-D). The overall structural organization of the JAK FERM is similar to canonical FERM domains and consists of three subdomains, the ubiquitin-like F1, acyl-CoA-binding protein-like F2, and pleckstrin homology domain-like F3, using the FERM domain terminology established for focal adhesion kinase (FAK) (48). These subdomains pack into a canonical trilobed FERM architecture (Figure 1A). While the overall domain topology is conserved when compared to FERM domains from the ezrin/radixin/moesin and FAK families, JAK FERMs posses several unique differences that enable an intimate interaction with the $\mathrm{SH} 2$ domain. First, an elongated linker, L1, connects the F1 and F2 domains and forms a large part of the interaction surface between the FERM and SH2 domains. In classical FERMs, L1 is typically between 13 and 15 residues but has extended to between 29 and 42 residues in JAKs. In addition to the L1, the $\mathrm{SH} 2$ domain packs against the F1- $\alpha 1$ helix, the highly conserved F3-SH2 linker (L2) and the SH2-pKD (L3) linker (44).

The F2 subdomain of the JAK FERM also deviates significantly from classical FERM domain structure. Additional residues in the linker between F2- $\alpha 1$ and F2- $\alpha 2$ allow for the extension of the N-terminal end of F2- $\alpha 2$ helix by one turn in JAK2 and two turns in TYK2 and JAK1 (Figure 2A). The C-terminal end of the F2- $\alpha 2$ and the N-terminal end of F2- $\alpha 3$ helices are also extended by one turn in JAK FERMs. The elongation of these two helices is likely related to the ability of the JAK FERM domains to utilize this interface to bind to cytokine receptors (discussed below). Additionally, the F2- $\alpha 3$ helix of JAKs is highly basic $(44,45)$, while in canonical FERM domains this chemical character is not preserved (49). The basic residues in this helix create a large positively charged patch on the JAKs, which may enable favorable interactions with the plasma membrane.

The core structure of the JAK F3 subdomain is highly similar to that of canonical FERM domains, consisting of a four-stranded $\beta$-sheet $(\beta 1-\beta 4)$ sandwiched against a three-stranded $\beta$-sheet $(\beta 5-\beta 7)$ followed by an $\alpha$-helix (F3- $\alpha 1)$ (Figure 2B). Yet interestingly, several loops within the F3 subdomain vary significantly between the JAK isoforms [for a comprehensive alignment of JAK family members, see Ref. (44)]. The first loop, between F3- $\beta 1$ and F3- $\beta 2$ strands, contains 12 amino acids in JAK2 and is 
A

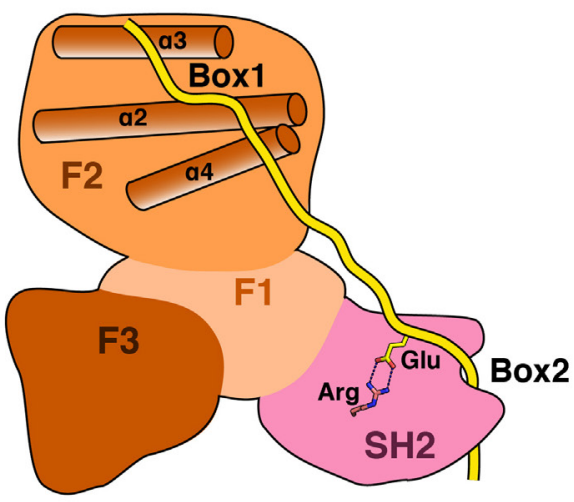

C

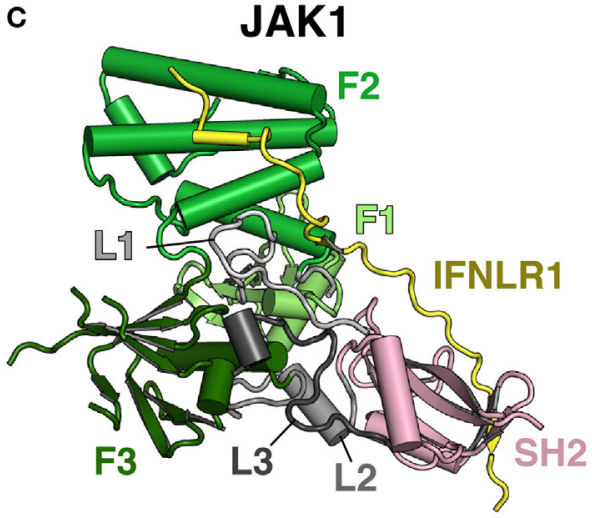

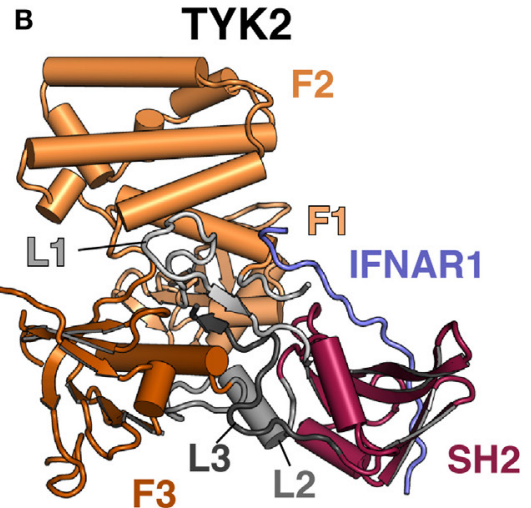

F3

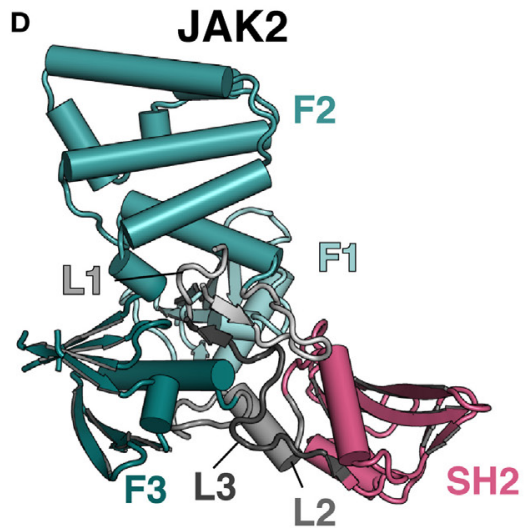

FIGURE 1 | Comparison of Janus kinase (JAK) FERM/SH2 domains. (A) Cartoon depiction of a JAK FERM and SH2 module bound to a receptor, illustrating receptor recognition principles discovered in recent crystal structures, followed by representations of crystal structures solved for (B) TYK2 (PDB ID 4PO6), (C) JAK1 (PDB ID 5L04), and (D) JAK2 (PDB ID 4Z32). The F1, F2, and F3 FERM subdomains are colored in various shades of orange, green, and blue, respectively. SH2 domains are colored in various shades of pink. Linkers L1, L2, and L3 are colored in various shades of gray. The IFNAR1 receptor peptide is colored blue, and the IFNLR1 is in yellow.
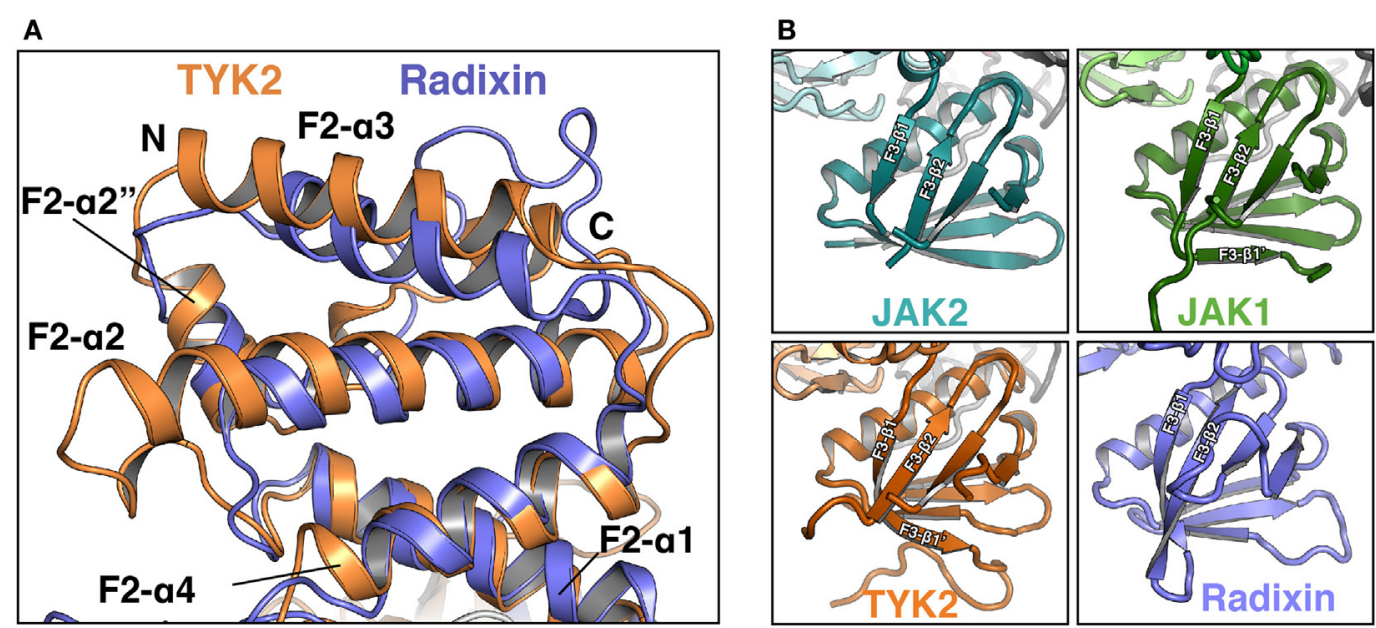

FIGURE 2 | Comparison of Janus kinase (JAK) and ERM family FERM domains. (A) Comparison of the FERM F2 subdomains of TYK2 (orange) and radixin (blue, PDB ID 1GC7). TYK2 and radixin F2 domains were superposed in PyMol with a root-mean-square deviation of $2.98 \AA$ A over 545 atoms. (B) Comparison of the F3 subdomains of JAK2 (top left, teal), JAK1 (top right, green), TYK2 (bottom left, orange), and radixin (bottom right, blue, PDB ID 1GC7). 
largely disordered in this crystal structure (Figure 2B, top left). In JAK1, the F3- $\beta 1 / \beta 2$ loop contains 22 amino acids and forms a $\beta$ hairpin that packs against F3- $\beta 7$, extending the $\beta$-sheet by a single strand, F3- $\beta 1^{\prime}$ (Figure 2B, top right). In TYK2, the loop is 35 amino acids and also forms a single strand that packs against F3- $\beta$ 7, with an additional loop visible (Figure 2B, bottom left). The C-terminal half of the linker in TYK2 is unstructured. The large insertions at this position are specific to JAK family FERM domains, as classical FERM domains contain only a short loop at this position (Figure 2B, bottom right). An additional disordered loop of variable length is located in between F3- $\beta 3$ and F3- $\beta 4$. F3- $\beta 3 / \beta 4$ linker lengths are 12,34 , and 44 amino acids in JAK2, JAK1, and TYK2, respectively. These insertions are also not present in canonical FERM domains. Interestingly, both the length and sequence identities of these JAK F3 insertions are highly conserved between all higher eukaryotic species.

The overall structure of the JAK family $\mathrm{SH} 2$ domain is reminiscent of a canonical phosphotyrosine (pTyr)-binding $\mathrm{SH} 2$ domain, consisting of a central $\beta$-sheet flanked by two $\alpha$-helices. In canonical SH2 domains, two loops flanking the $\mathrm{SH} 2-\alpha \mathrm{B}$ helix form a hydrophobic groove that is the binding site for specificity determining residues at positions +3 and +5 relative to the $\mathrm{pTyr}$ (50). In the JAK SH2 domain, the SH2-EF loop and a $\beta$-hairpin formed by SH2- $\beta \mathrm{G} 1$ and $\mathrm{SH} 2-\beta \mathrm{G} 2$ create a large hydrophobic slot that is key for receptor Box 2 binding (discussed below). In addition, the conserved, phosphate-coordinating arginine residue located at the base of the pTyr binding pocket is conserved in all JAKs except TYK2, where it has been replaced with a histidine residue.

\section{THE STRUCTURE OF TYK2 BOUND TO IFNAR1 BOX2}

Structural studies of the JAKs bound to their receptors have historically been hampered by poor expression and solubility of the JAKs as well as weak affinities for their receptors. To overcome these biochemical hurdles, a construct containing a juxtamembrane peptide from IFNAR1 fused to the C-terminus of the TYK2 FERM and SH2 domains was used to generate the TYK2-IFNAR1 complex (44). Prior studies had shown that IFNAR1 is not trafficked to the cell surface in the absence of TYK2 (51), suggesting a strong constitutive interaction between these two molecules. This approach greatly enhanced the expression and solubility of the TYK2 FERM-SH2 and enabled crystallization of TYK2 with a peptide encompassing the Box2 sequence (residues 478-512) of IFNAR1 (44). Importantly, this structure revealed the Box2 of IFNAR1 bound in an extended conformation to a composite interface on the surface of the TYK2 FERM-SH2, with the bulk of the peptide interacting with the $\mathrm{SH} 2$ domain (Figure 3A).

The overall topology of the IFNAR1 Box2 interaction with TYK2 resembles that of a pTyr-containing peptide bound to an $\mathrm{SH} 2$ domain and can be broken down into three sections, all of which are important for stable interaction with TYK2 (44). At the N-terminus of the Box2 motif, triplet leucine residues, Leu490, Leu491, and Leu492, participate in a key interaction by packing against a hydrophobic groove formed between F2, L1, and F1 (Figure 3A, top panel). The next segment of IFNAR1 binds to what would be the pTyr binding pocket in a canonical $\mathrm{SH} 2$ domain (Figure 3A, middle panel). However, in place of a pTyr residue, a conserved glutamate (Glu497) is placed in the pocket. In TYK2, the arginine residue responsible for forming a salt bridge with the phosphate group in classical $\mathrm{SH} 2$ domain interactions is replaced with a histidine residue (His474), which does not participate in the interaction with IFNAR1. Instead, the sidechain carboxylate of Glu497 forms hydrogen bond interactions with residues Ser476 and Thr477 of TYK2, mimicking a subset of the contacts normally made by the phosphate group (Figure 3A, middle panel). TYK2 is unique among human JAKs, as all other family members maintain an arginine at this position, although mutagenesis of this Arg has been shown to not affect subcellular localization or function (52). In the third segment, the hydrophobic Box2 sequence of IFNAR1 forms a short $\beta$ sheet with the $\beta$ G1 strand of the TYK2 SH2 domain (Figure 3A, bottom panel). Two hydrophobic residues located at the +5 and +7 positions relative to Glu497, Cys502 and Ile504, pack into the hydrophobic groove formed between the SH2-EF loop and the $\mathrm{SH} 2-\beta \mathrm{G} 1 / 2$ hairpin. Importantly, this interaction is primarily mediated by backbone $\mathrm{H}$-bond and aliphatic sidechain van der Waals interactions, therefore revealing how JAK SH2 domains can bind a range of Box 2 sequences that contain aliphatic residues in the first and third positions.

\section{THE JAK1 FERM DOMAIN INTERACTION WITH BOX1 SEQUENCES FROM CLASS II RECEPTORS}

The structure of TYK2 bound to IFNAR1 only revealed the binding site for residues near the Box2 peptide; therefore, further structural characterization was required to elucidate the JAK binding site for the Box1 motif. To this end, crystal structures of the JAK1 FERM-SH2 bound to the Box1 of IFNLR1 and IL10R1 were determined (45), utilizing a co-expression and screening approach to identify tight binding receptors amenable to crystallization. Rather than expressing the FERM-SH2 and receptor as a fusion as with TYK2 and IFNAR1, the JAK1 FERM-SH2 and receptor were co-expressed as His and GST tag fusion proteins, respectively. This method identified IFNLR1 and IL10R1 as receptors that bound with high enough affinity to enable copurification of a JAK-receptor complex that could be utilized in crystallization trials (45).

Importantly, these structures revealed a novel binding site for the Box1 motif of class II cytokine receptors. The Box1 of IFNLR1 binds to the F2 subdomain of the JAK1 FERM, in a cleft formed by helices F2- $\alpha 2, \mathrm{~F} 2-\alpha 3$, and F2- $\alpha 4$ (Figure 3B). Interestingly, the location of the Box1 binding site was previously hypothesized, based on CONSURF-predicted sequence conservation within that region (53) and the effects of F2 destabilization on receptor binding (54). Like the IFNAR1 Box2 peptide interaction with TYK2, the IFNLR1 Box1 interface with JAK1 can be subdivided into multiple distinct interaction sites. The first is defined by Pro256, Trp257, and Phe258, which fold into a $3_{10}$ helix that facilitates the insertion of Trp 257 into a deep groove formed by the F2- $\alpha 2$ and 


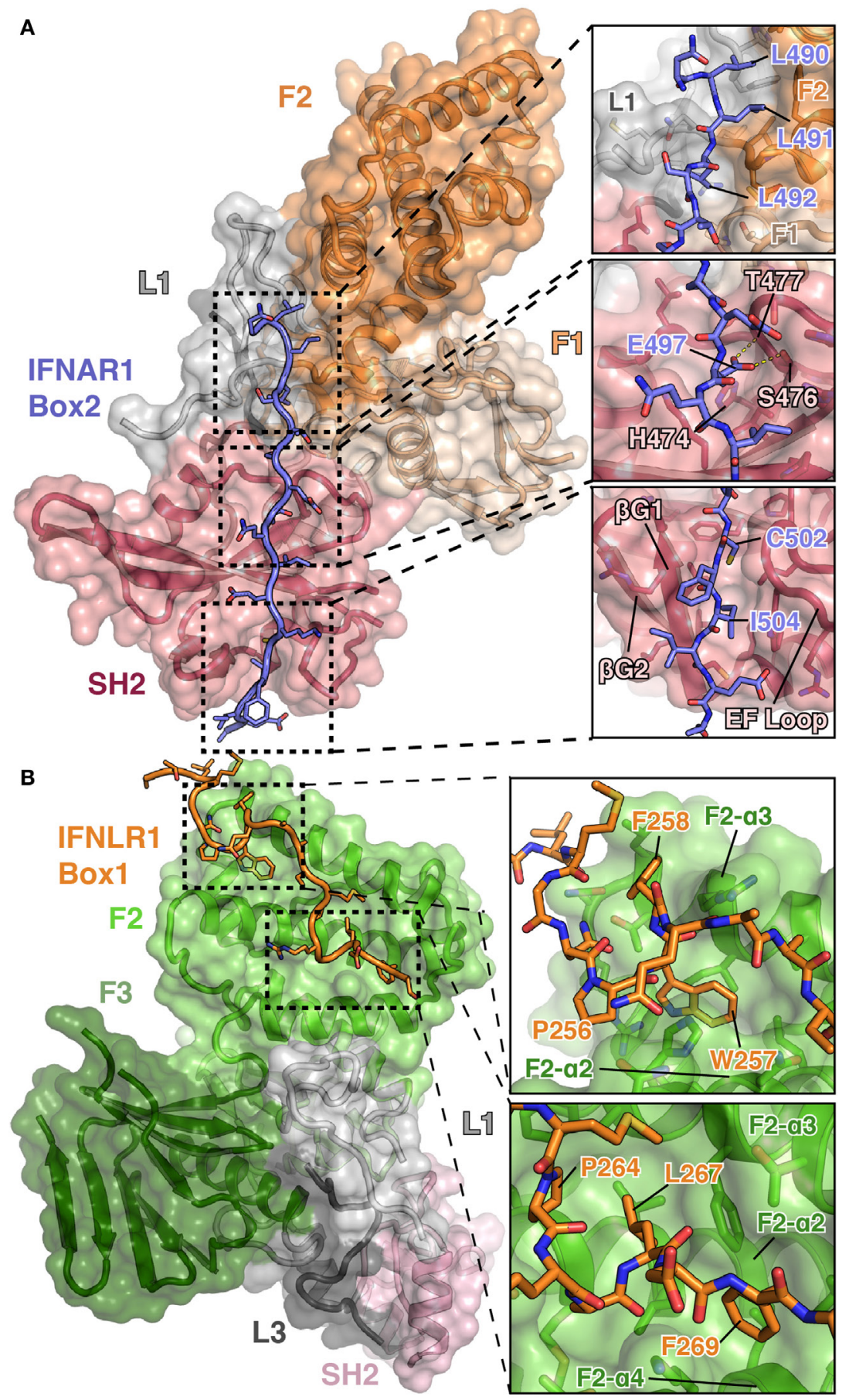

FIGURE 3 | Detailed views of the interactions between TYK2-IFNAR1 Box2 and JAK1 IFNLR1 Box1. (A) Overview of the interaction between TYK2 FERM and SH2 (colored as in Figures 1A,B) domains in complex with the IFNAR1 Box2 region (left, PDB ID 4PO6). Detailed interactions are shown for three regions of the TYK2-IFNAR1 complex (right panels). In the N-terminal tri-leucine region, Leu490, Leu491, and Leu492 of IFNAR1 pack against a hydrophobic groove between the TYK2 F1 (tan), L1 (gray), and F2 (orange) domains (top panel). In the center of the interaction, Glu497 of IFNAR1 forms hydrogen bonds with Ser476 and Thr477 (middle panel). At the C-terminus of IFNAR1, Cys502 and lle504 insert into a groove between the $\beta$-G1/2 hairpin and EF loop of the TYK2 SH2 domain (bottom panel). (B) Overview of the bipartite interaction between JAK1 FERM and SH2 (colored as in Figure 1C) domains in complex with the IFNLR1 Box1 region (left, 5IXD). Detailed interactions are shown for two regions of the JAK1-IFNLR1 complex (right panels). At the first site, IFNLR1 residue Trp257 inserts itself into a groove between JAK1 F2- $\alpha 2$ and F2- $\alpha 3$ (top panel). At the second site, hydrophobic residues Pro264, Leu267, and Phe269 of IFNLR1 pack into a groove formed by JAK1 F2- $\alpha 2, F 2-\alpha 3$, and F2- $\alpha 4$ (bottom panel). 
$\alpha 3$ helices (Figure 3B, top panel). Following Phe258, the IFNLR1 Box1 breaks contact with JAK1, rejoining at a second site defined by the motif PxxLxF (45). This motif folds into a second $3_{10}$ helix, aligning Pro264, Leu267, and Phe269 into a hydrophobic ridge that interacts with a groove formed by the F2- $\alpha 2, \alpha 3$, and $\alpha 4$ helices in the JAK1 FERM domain (Figure 3B, bottom panel). The proline and leucine residues found in this PxxLxF motif are completely conserved in all type II cytokine receptors, while the phenylalanine is a favored residue (Figure 4) (45). Indeed, in a second structure of a hybrid receptor formed by an N-terminal fusion of IFNLR1 "PWF" motif with the IL10R1 Box1 "PxxLxF" and Box2 motifs, the IL10R1 Box1 sequence (PSVLLF) bound in a nearly identical conformation to that seen for IFNLR1 (45).

Unlike TYK2, the JAK1 FERM-SH2 domain was soluble when purified in the absence of a receptor, enabling biophysical studies of receptor binding and granular dissection of the Boxl interaction. Quantitative biophysical measurement of the interaction between JAK1 and IFNLR1 peptide containing both Box1 and Box 2 by Biolayer Interferometry established a binding affinity $\left(K_{\mathrm{D}}\right)$ of $70 \mathrm{nM}$. The isolated IFNLR1 Box1 binds to JAK1 with an affinity of $1.2 \mu \mathrm{M}$, while the isolated Box 2 does not measurably interact (45). These results indicate that while both Box1 and Box 2 contribute to the JAK-IFNLR1 interaction, Box1 is the primary driver of the interaction. At this point, it is unknown if this observation will apply to interaction between other JAKs and receptors.

\section{STRUCTURE OF JAK1 BOUND TO IFNLR1 BOX1 AND BOX2}

An important second crystal structure of the JAK1 FERM-SH2 bound to a peptide containing both the Box1 and Box2 domains of IFNLR1 has also been published (Figure 5A) (46). This structure, which was produced using the JAK1 FERM-SH2 linked to the IFNLR1 peptide at the C-terminus, for the first time demonstrates simultaneous binding of both Box 1 and Box 2 sites to a single JAK monomer. The model obtained from this structure confirms both the Box1 interaction site identified for JAK1 as well as authenticates key conserved features of the Box2 interaction identified in the TYK2-IFNAR1 structure (44). In addition, it corroborates the affinity measurements that indicate IFNLR1 Box 1 and Box 2 cooperate to create a high affinity 1:1 interaction with JAK1 (45).

As mentioned above, the structure of the IFNLR1 Box1 region is nearly identical to that of previously solved structures of JAK1IFNLR1 and JAK1-IL10R1 Box1 peptides (45). Five additional linker residues that bridge the Box 1 and Box 2 regions are also present in the structure, although the electron density is relatively weak for this region due to a lack of peptide contacts with JAK1. More importantly, the key features of the JAK1-IFNLR1 Box2 region are conserved to the TYK2-IFNAR1 structure, yet with several notable differences (Figure 5B). First and foremost, like IFNAR1, IFNLR1 also contains a glutamate residue (Glu285)

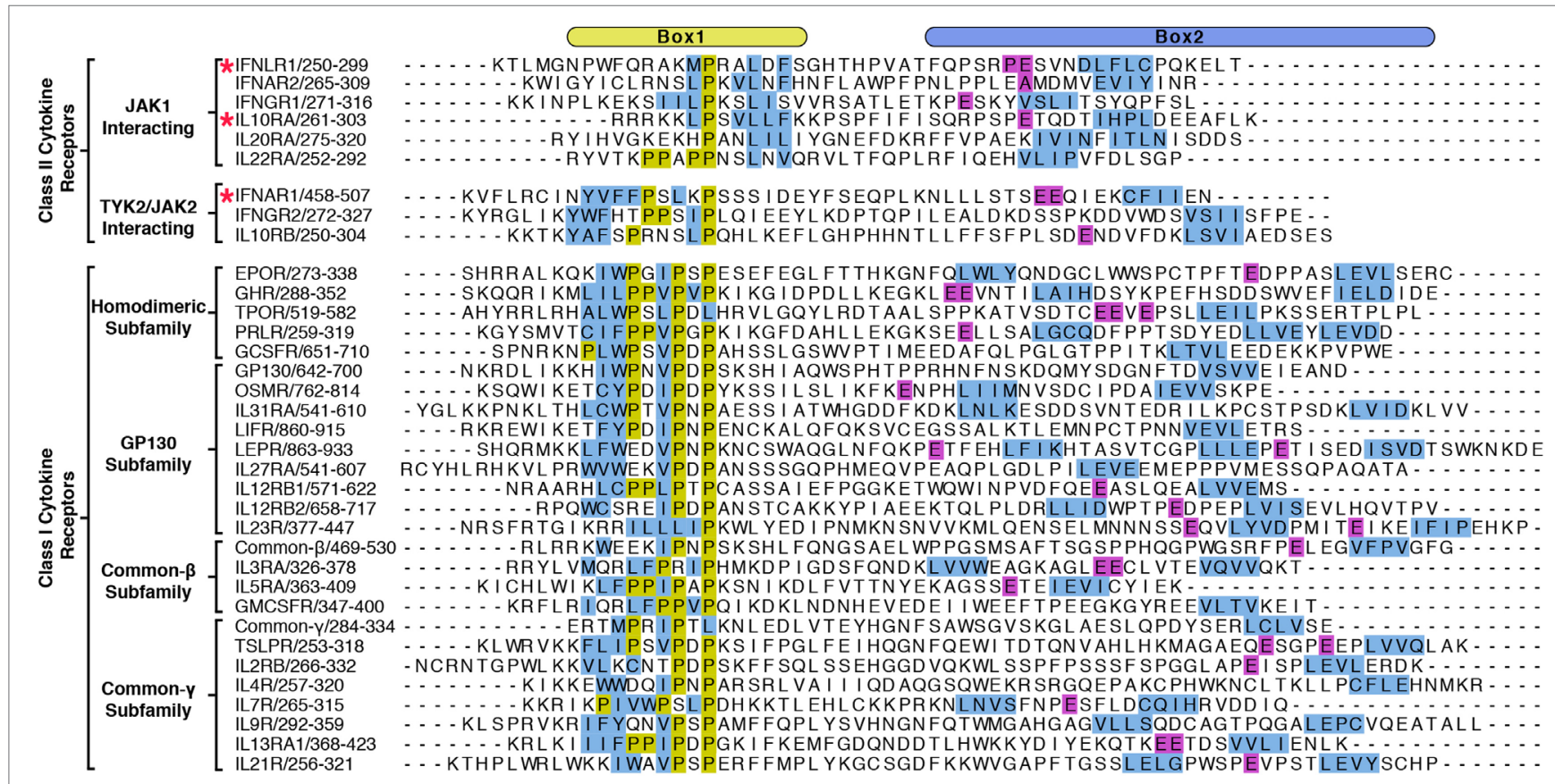

FIGURE 4 | Alignment of Box1 and Box2 motifs from Janus kinase (JAK)-interacting cytokine receptors. The juxtamembrane intracellular sequences from all known signaling receptors that interact with JAKs were aligned by their Box 1 proline motifs. Receptors are grouped by class I and class II, followed by subgroupings by either the JAK they interact with (class II) or by subfamily (class I). Due to the low sequence homology between receptors, the alignments were produced by hand, and the residues colored in order to highlight some of the shared features of the receptors. Hydrophobic residues within the Box 1 region are colored in blue and prolines in yellow. For the Box2 region, putative four-residue hydrophobic motifs (aliphatic-X-aliphatic-X) are colored in blue, with glutamate residues three to seven residues N-terminal to these motifs are colored in purple. Receptors with known structures (IFNAR1, IFNLR1, and IL10RA) are marked with a star. 


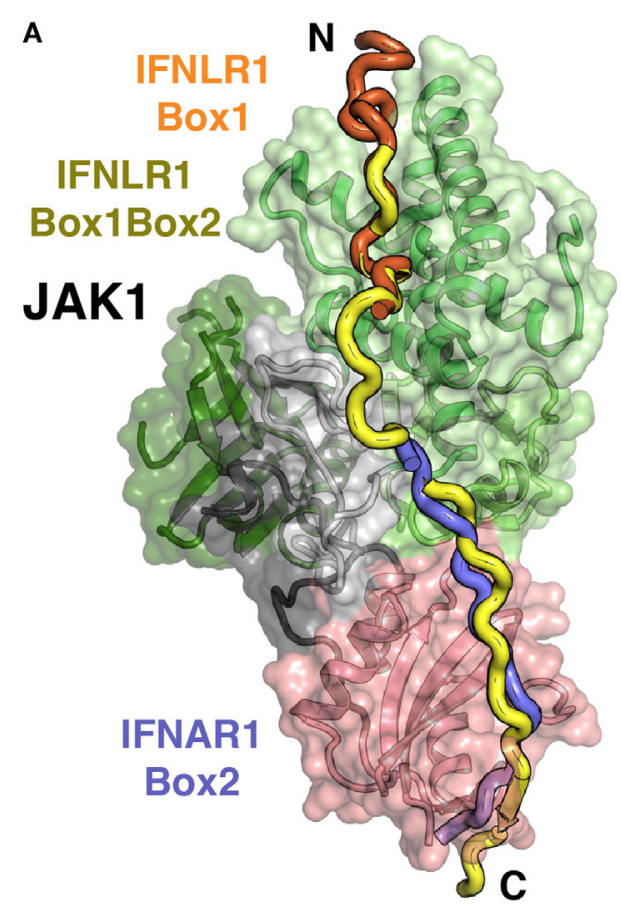

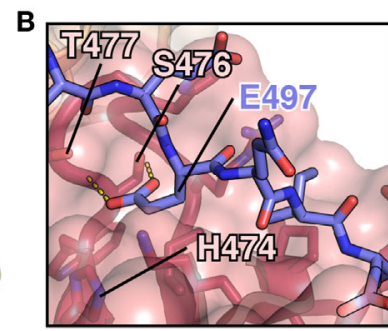

TYK2/IFNAR1

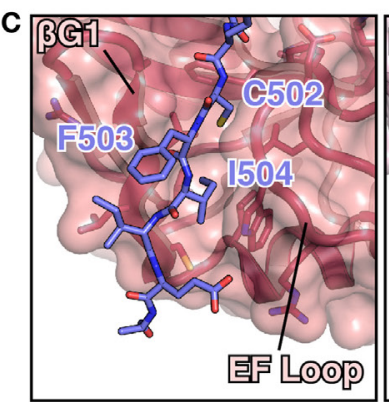

TYK2/IFNAR1

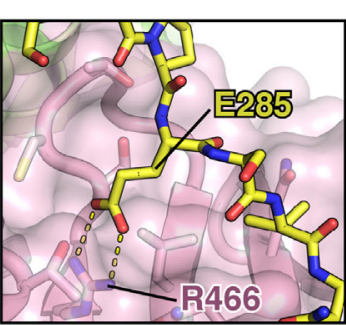

JAK1/IFNLR1

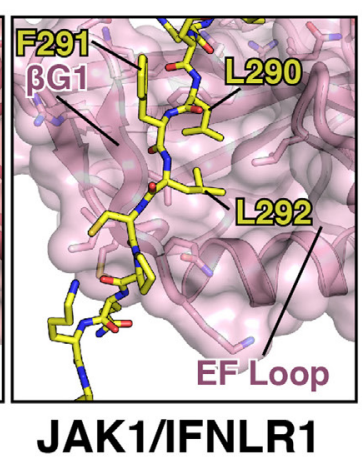

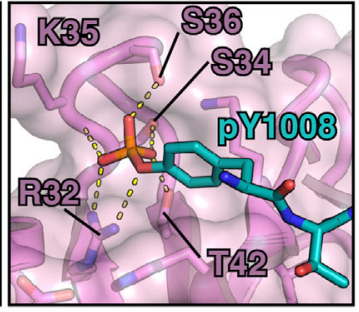

SHP-2/Pdgfrb

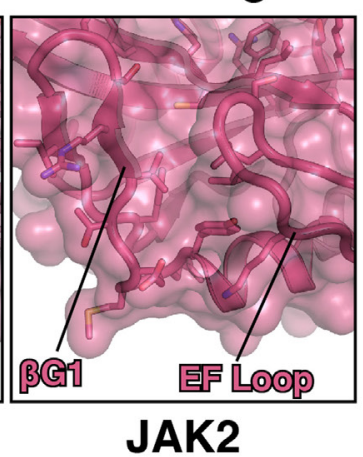

FIGURE 5 | JAK1 and TYK2 recognize the Box2 motif via a conserved mechanism. (A) Cartoon representation of JAK1 (colored as in Figure 1C) bound to IFNLR1 receptor peptide (yellow) containing both the Box1 and Box2 motifs (PDB ID 5L04). For comparison, the IFNLR1 Box1 motif (orange) and the IFNAR1 Box2 (blue) are shown after superposition of JAK1 and TYK2, respectively. (B) Detailed comparison of the pTyr/Glu binding pocket of the TYK2 (PDB ID 4PO6), JAK1, and SHP-2 (PDB ID 1AYA) SH2 domains. TYK2 (left) contains a histidine residue (H474) at the base of the pocket, while JAK1 and SHP-2 have an arginine at this position that forms a salt bridge with either the glutamate in IFNLR1 or phosphate group in the PDGF receptor peptide. (C) Detailed comparison of the Box2 binding groove of TYK2, JAK1, and JAK2. IFNAR1 residues Cys502 and lle504 pack into a groove in the TYK2 SH2 domain between the $\beta$-G1/2 hairpin and EF loop (left). In the JAK1/IFNLR1 structure, Leu290 and Leu292 pack into this groove (center). The SH2 domain of JAK2 (PDB ID $4 Z 32$ ) maintains this conformation in the absence of receptor (right).

that extends into the SH2-pTyr binding pocket (Figure 5B). Yet unlike in TYK2, the canonical pTyr binding SH2 arginine residue (Arg466) in JAK1 forms a salt bridge interaction with Glu285. This closely approximates the interaction between the $\mathrm{SH} 2$ arginine and phosphate group in a canonical SH2-pTyr interaction, represented here by the SHP-2/Pdgfrb complex structure (Figure 5B). This interaction, while slightly different in topology to the TYK2-IFNAR1 Glu497 interaction, further supports the hypothesis that the JAK family $\mathrm{SH} 2$ domain evolved from an archaic precursor that participated in conditional receptor interactions regulated by phosphorylation (44).

Similarities between JAK1 and TYK2 peptide recognition extend to other sites identified in the TYK2-IFNAR1 interaction. The key "segment 1" leucine triplet found in IFNAR1 is changed to an Ala-Thr-Phe sequence in IFNLR1, yet it maintains a similar binding orientation. IFNLR1 Ala277 and Thr278 pack into the F2/L1 pocket in a similar conformation to IFNAR1 Leu490 and Leu491, while Leu492 of IFNAR1 is replaced by Phe279 in IFNLR1 and binds to the same pocket at the interface of the F1 and $\mathrm{SH} 2$ domains. While these binding triplets have a similar interaction phenotype, the gap between the IFNAR1 Leu492/ IFNLR1 Phe279 residue and the SH2-interacting glutamate is six residues in IFNLR1 rather than the five residues seen in IFNAR1.
This has profound effects on the backbone positioning of the peptide, which bulges out to accommodate the extra amino acid (46).

Additionally, the interaction between the hydrophobic Box2 and $\mathrm{SH} 2$ domain is conserved in the JAK1-IFNLR1 structure (Figure 5C). Like IFNAR1, the Box2 motif in IFNLR1 begins five residues C-terminal to the conserved glutamate (Glu285), with the +5 and +7 residues (Leu290 and Leu292) buried in a groove formed by the SH2-EF loop and $\beta$ G1 strand. The Box 2 motif also caps an antiparallel sheet formed with the $\beta \mathrm{G} 1$ and $\beta \mathrm{G} 2$ strands via a number of backbone hydrogen bonds, in a similar manner to that seen for the IFNAR1 Box2 peptide (44). This further reinforces the hypothesis that Box 2 interactions are primarily driven by main chain Box $2-\beta \mathrm{G} 1$ sheet formation and burial of aliphatic sidechains into the hydrophobic core of the SH2 domain (44).

\section{INSIGHTS INTO THE BASIS OF JAK-RECEPTOR SPECIFICITY}

The elucidation of FERM-SH2 structures from three of the four JAK family members enables the comparison of receptor-binding surfaces of each JAK (Figure 6A). Sequence analysis of the receptor-binding interfaces on the FERM F2 and $\mathrm{SH} 2$ domains 
indicates a significant level of conservation among JAK family members, suggesting that all JAKs utilize the two common interfaces described here to interact with their receptors (Figure 6A) $(44,53,55)$. While we are far from a complete understanding of key binding determinants of all JAK-receptor interactions, there are several differences between the JAK FERM F2 subdomains that may create incompatibility with certain Boxl receptor sequences. The most obvious example of this can be seen in comparison of the positioning of the F2- $\alpha 3$ helix between JAK1, TYK2, and JAK2. The F2- $\alpha 3$ helix conformation is conserved

A
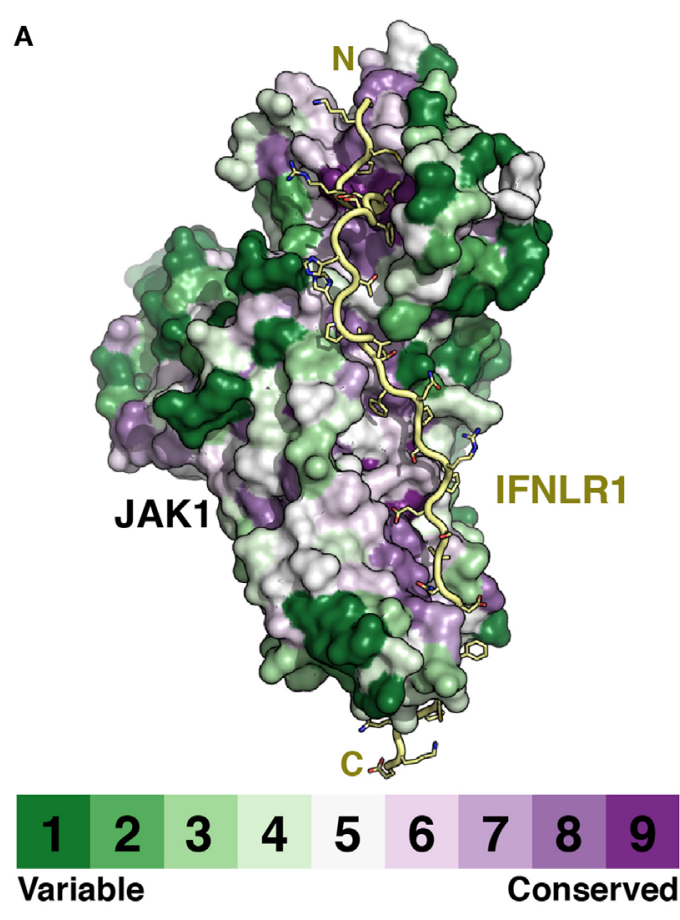

B

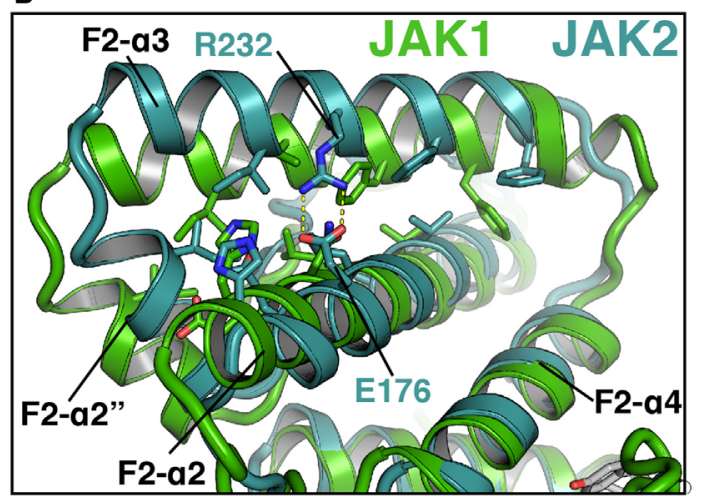

FIGURE 6 | Janus kinases (JAKs) use conserved surfaces to mediate receptor interactions. (A) CONSURF analysis (55) of a ClustalO alignment of 245 JAK homologs projected onto the surface of JAK1 (PDB ID 5L04). Less conserved residues are colored in green, highly conserved residues are colored purple. IFNLR1 (yellow) shown to illustrate highly conserved residues at receptor-binding surface. (B) Superposition of JAK1 (green, PDB ID 5IXD) and JAK2 (cyan, PDB ID 4Z32) with a root-mean-square deviation of $1.17 \AA$ over 1,119 atoms to illustrate shift in position of the F2- $\alpha 3$ and F2- $\alpha 2$ " helices in JAK2. between all JAK1 and TYK2 structures available thus far, while the JAK2 F2- $\alpha 3$ deviates significantly (Figure 6B). A rotation of the JAK2 F2- $\alpha 3$ results in a shift in the N-terminus of the helix, allowing the formation of a salt bridge between Arg232 and Glu176 and an inward translation of the F2- $\alpha 2$ " helix. These conformational changes close off the hydrophobic pocket that, in JAK1, occupies W257 of IFNLR1. In addition to this change, differences in the linker and F2- $\alpha 2$ helix length observed between JAK1, TYK2, and JAK2 (Figure 1) along with sidechain variations within the peptide binding F2- $\alpha 2 / \alpha 3$ interface suggest that the Box 1 conformation seen for IFNLR1 and IL10RA bound to JAK1 may be specific to JAK1-class II receptor interactions. In other words, the class II PxxLxF sequence may be a bona fide JAK1-specific binding motif (Figure 4). Importantly, a different, conserved Box1 PxP motif has been found to be critical for JAK binding and functional signaling in various receptors, including gp130 (15), GHR (56), G-CSFR (57), and EPOR (28) and can seemingly interact with both JAK1 and JAK2 (Figure 4) (58). It is possible that the $\mathrm{PxP}$ motif (along with surrounding residues) has evolved to be a more degenerate JAK binding motif, which could explain the ability of some receptors to seemingly interact with multiple JAKs (58). Further structures of JAK1, TYK2, and/ or JAK2 bound to Box1 receptor fragments will be required to answer the question of specificity versus degeneracy in JAK-Box 1 interactions. Regardless, comparison of these structures suggests that the F2 subdomain is a dynamic interaction domain that can bind multiple receptor peptides, each potentially adopting a distinct conformation.

With regards to the $\mathrm{SH} 2$ domain, the two structures that define our understanding of SH2-Box2 interactions (TYK2-IFNAR1 and JAK1-IFNLR1) show a remarkable level of conservation in their interaction mechanisms given the low sequence homology between receptors (Figure 4). Both receptors utilize glutamate residues to interact with the vestigial pTyr binding pocket and aliphatic Box 2 motifs to cap a $\beta$-hairpin in the $\mathrm{SH} 2$ domain. Importantly, in the apo structure of JAK2, the Box2 binding groove is open and appears to be competent for Box2 binding (Figure 5C, right panel). Therefore, with the examples in hand, it seems likely that the Box 2 interaction mechanism is conserved across all JAK-receptor interactions. What is still unknown is how much specificity the Box 2 brings to a JAK-receptor interaction, given the degenerate nature of Box2 interactions via main chain hydrogen bonding and aliphatic contacts.

\section{CONCLUSION AND PERSPECTIVES}

In recent years, significant progress has been made in our understanding of the structure of JAK kinases. In addition to structures of the FERM-SH2 modules and the mechanism of interaction with the receptors described in this review, structural models for the pseudokinase-kinase modules from TYK2 and JAK2 have also been revealed, providing an explanation for the mechanism of negative regulation by the pseudokinase as well as mutations linked to myeloproliferative diseases present in the $\operatorname{psKD}(35,36)$. Additionally, the structure of the JAK2 kinase domain bound to its negative regulator SOCS3 has provided details on the regulation 
of the JAK-STAT pathway (59). Yet, despite these advances, many outstanding questions remain with regards to JAK FERM-SH2 domains and their interacting receptors. One major question is whether the FERM-SH2 has a role in activation of the kinase domain upon cytokine binding. For example, the dimerization of FERM domains from FAK has been shown to be essential to activation (60). FAK, like the JAKs, is a multi-domain tyrosine kinase containing a FERM and a kinase domain. The FAK FERM domain interacts directly with the kinase domain to mediate inhibition (61), and proximity-induced auto-phosphorylation and activation of FAK is dependent on its dimerization (60). Given that dimerization is a prerequisite to JAK activation, a role for the FERM-SH2 module in kinase activation is an intriguing possibility.

A second question is what role the enormous variation in cytokine receptor intracellular sequences plays in JAK activation (Figure 4). Even the earliest papers noted the lack of obvious consensus sequences among the intracellular domains of cytokine receptors (15). With several JAK-receptor structures now known we understand more about how receptors interact with JAKs, but it is still unclear why there is so much variation in JAK-interacting sequences. What does seem clear is that this variation is important and likely underlies important differences in JAK-receptor affinity and conformation that affects kinase activation and subsequent downstream signaling. For example, tryptophan residues within the "interbox" region (between Box1 and Box2) have been shown to disrupt JAK1 interaction and dominantly disrupt kinase signaling (26, 62-64). Yet, these Trp residues, while conserved within gp130 and EPOR isoforms, are not in the same position in relation to one another or conserved to other cytokine receptors (Figure 4). Mutations upstream of the Box1 sequence in gp130 and EPOR have also been identified that disrupt JAK signaling, but not receptor binding $(65,66)$. This result suggests a role for these membrane proximal sequences in facilitating proper JAK positioning to enable kinase activation. Yet, these obviously important residues are also not well conserved among receptors (Figure 4). While many questions remain unanswered, each set

\section{REFERENCES}

1. Leonard WJ, O'Shea JJ. Jaks and STATs: biological implications. Annu Rev Immunol (1998) 16:293-322. doi:10.1146/annurev.immunol.16.1.293

2. Schwartz DM, Bonelli M, Gadina M, O'Shea JJ. Type I/II cytokines, JAKs, and new strategies for treating autoimmune diseases. Nat Rev Rheumatol (2016) 12:25-36. doi:10.1038/nrrheum.2015.167

3. Brooks AJ, Dai W, O’Mara ML, Abankwa D, Chhabra Y, Pelekanos RA, et al. Mechanism of activation of protein kinase JAK2 by the growth hormone receptor. Science (2014) 344:1249783. doi:10.1126/science.1249783

4. Moraga I, Wernig G, Wilmes S, Gryshkova V, Richter CP, Hong WJ, et al. Tuning cytokine receptor signaling by re-orienting dimer geometry with surrogate ligands. Cell (2015) 160:1196-208. doi:10.1016/j.cell.2015.02.011

5. Ghoreschi K, Laurence A, O'Shea JJ. Janus kinases in immune cell signaling. Immunol Rev (2009) 228:273-87. doi:10.1111/j.1600-065X.2008.00754.X

6. Haan C, Kreis S, Margue C, Behrmann I. Jaks and cytokine receptors - an intimate relationship. Biochem Pharmacol (2006) 72:1538-46. doi:10.1016/j. bcp.2006.04.013

7. Quintás-Cardama A, Kantarjian H, Cortes J, Verstovsek S. Janus kinase inhibitors for the treatment of myeloproliferative neoplasias and beyond. Nat Rev Drug Discov (2011) 10:127-40. doi:10.1038/nrd3264 of unique receptor sequences undoubtedly encode a finely tuned level of kinase activity that is turned on in response to ligand binding, allowing JAK pathways to work effectively without inducing deleterious effects like cancer and autoimmunity.

The JAKs are not unique in their utilization of a FERM domain to mediate partner interactions. The FERM domain is a remarkably prolific protein interaction module, having evolved at least four separate interaction sites for both proteins and lipids. The FERM F3 subdomain, in particular, utilizes multiple surfaces to interact with a wide variety of binding partners. The ERM family members radixin and merlin along with myosin $\mathrm{X}$ use a shallow groove between F3- $\beta 5$ and F3- $\alpha 1$ to interact with their partner molecules (67-70), while radixin has an additional peptide binding site formed by a pocket between F3- $\beta 4$ and F3- $\beta 7$ as well as a lipid interaction site between the F2 and F3 subdomains (71, 72). Additionally, myosin VIIa has been shown to use a composite interface at the junction of F1, F2, and F3 to bind to the scaffold protein SANS (73). The F2 subdomain is also involved in a number of FERM-peptide interactions. Most notably, the tumor suppressor protein merlin binds to its interaction partners LATS1/2 via its F2 domain (74). LATS1/2 bind to the same F2- $\alpha 2$, $\alpha 3$, and $\alpha 4$ groove that the IFNLR1 and IL10RA Box1 motifs were found to bind, although with opposite directionality. Merlin and moesin also contain an inhibitory CTD that binds to the FERM using a combination of the F2 and F3- $\beta 4 / \beta 7$ sites $(49,74)$. While the evolutionary history of JAK FERM-receptor interactions will likely remain a mystery, it is tempting to speculate that an ancestral SH2-containing JAK recombined with a FERM domain that eventually facilitated conversion to a constitutive JAKreceptor interaction. Regardless, the conformational similarities and differences highlighted here reinforce the impressive plasticity and modularity of FERM and SH2 domains, both in the JAKs as well as other vital membrane-associated signaling molecules.

\section{AUTHOR CONTRIBUTIONS}

RF and PL wrote and edited the manuscript.

8. de Vos AM, Ultsch M, Kossiakoff AA. Human growth hormone and extracellular domain of its receptor: crystal structure of the complex. Science (1992) 255:306-12. doi:10.1126/science. 1549776

9. Bazan JF. Structural design and molecular evolution of a cytokine receptor superfamily. Proc Natl Acad Sci U S A (1990) 87:6934-8. doi:10.1073/pnas.87. 18.6934

10. Wang X, Lupardus P, Laporte SL, Garcia KC. Structural biology of shared cytokine receptors. Annu Rev Immunol (2009) 27:29-60. doi:10.1146/annurev. immunol.24.021605.090616

11. Watowich SS, Hilton DJ, Lodish HF. Activation and inhibition of erythropoietin receptor function: role of receptor dimerization. Mol Cell Biol (1994) 14:3535-49. doi:10.1128/MCB.14.6.3535

12. Renauld J-C. Class II cytokine receptors and their ligands: key antiviral and inflammatory modulators. Nat Rev Immunol (2003) 3:667-76. doi:10.1038/ nri1153

13. Skiniotis G, Lupardus PJ, Martick M, Walz T, Garcia KC. Structural organization of a full-length gp130/LIF-R cytokine receptor transmembrane complex. Mol Cell (2008) 31:737-48. doi:10.1016/j.molcel.2008.08.011

14. Fukunaga R, Ishizaka-Ikeda E, Pan CX, Seto Y, Nagata S. Functional domains of the granulocyte colony-stimulating factor receptor. EMBO J (1991) 10:2855-65. 
15. Murakami M, Narazaki M, Hibi M, Yawata H, Yasukawa K, Hamaguchi M, et al. Critical cytoplasmic region of the interleukin 6 signal transducer gp 130 is conserved in the cytokine receptor family. Proc Natl Acad Sci U S A (1991) 88:11349-53. doi:10.1073/pnas.88.24.11349

16. O'Neal KD, Yu-Lee LY. The proline-rich motif (PRM): a novel feature of the cytokine/hematopoietin receptor superfamily. Lymphokine Cytokine Res (1993) 12:309-12.

17. Krolewski JJ, Lee R, Eddy R, Shows TB, Dalla-Favera R. Identification and chromosomal mapping of new human tyrosine kinase genes. Oncogene (1990) 5:277-82.

18. Takahashi T, Shirasawa T. Molecular cloning of rat JAK3, a novel member of the JAK family of protein tyrosine kinases. FEBS Lett (1994) 342:124-8. doi:10.1016/0014-5793(94)80485-0

19. Harpur AG, Andres AC, Ziemiecki A, Aston RR, Wilks AF. JAK2, a third member of the JAK family of protein tyrosine kinases. Oncogene (1992) 7:1347-53.

20. Wilks AF, Harpur AG, Kurban RR, Ralph SJ, Zürcher G, Ziemiecki A. Two novel protein-tyrosine kinases, each with a second phosphotransferase-related catalytic domain, define a new class of protein kinase. Mol Cell Biol (1991) 11:2057-65. doi:10.1128/MCB.11.4.2057

21. Müller M, Briscoe J, Laxton C, Guschin D, Ziemiecki A, Silvennoinen O, et al. The protein tyrosine kinase JAK1 complements defects in interferonalpha/beta and -gamma signal transduction. Nature (1993) 366:129-35. doi:10.1038/366129a0

22. Velazquez L, Fellous M, Stark GR, Pellegrini S. A protein tyrosine kinase in the interferon alpha/beta signaling pathway. Cell (1992) 70:313-22. doi:10.1016/0092-8674(92)90105-L

23. Argetsinger LS, Campbell GS, Yang X, Witthuhn BA, Silvennoinen O, Ihle JN, et al. Identification of JAK2 as a growth hormone receptor-associated tyrosine kinase. Cell (1993) 74:237-44. doi:10.1016/0092-8674(93)90415-M

24. Lütticken C, Wegenka UM, Yuan J, Buschmann J, Schindler C, Ziemiecki A, et al. Association of transcription factor APRF and protein kinase Jak1 with the interleukin-6 signal transducer gp130. Science (1994) 263:89-92. doi:10.1126/science.8272872

25. Colamonici OR, Uyttendaele H, Domanski P, Yan H, Krolewski JJ. p135tyk2, an interferon-alpha-activated tyrosine kinase, is physically associated with an interferon-alpha receptor. J Biol Chem (1994) 269:3518-22.

26. Witthuhn BA, Quelle FW, Silvennoinen O, Yi T, Tang B, Miura O, et al. JAK2 associates with the erythropoietin receptor and is tyrosine phosphorylated and activated following stimulation with erythropoietin. Cell (1993) 74:227-36. doi:10.1016/0092-8674(93)90414-L

27. Miyazaki T, Kawahara A, Fujii H, Nakagawa Y, Minami Y, Liu ZJ, et al. Functional activation of Jak1 and Jak3 by selective association with IL-2 receptor subunits. Science (1994) 266:1045-7. doi:10.1126/science.7973659

28. Huang LJ, Constantinescu SN, Lodish HF. The N-terminal domain of Janus kinase 2 is required for Golgi processing and cell surface expression of erythropoietin receptor. Mol Cell (2001) 8:1327-38. doi:10.1016/ S1097-2765(01)00401-4

29. Frank SJ, Yi W, Zhao Y, Goldsmith JF, Gilliland G, Jiang J, et al. Regions of the JAK2 tyrosine kinase required for coupling to the growth hormone receptor. J Biol Chem (1995) 270:14776-85. doi:10.1074/jbc.270.24.14776

30. Zhao Y, Wagner F, Frank SJ, Kraft AS. The amino-terminal portion of the JAK2 protein kinase is necessary for binding and phosphorylation of the granulocyte-macrophage colony-stimulating factor receptor beta c chain. J Biol Chem (1995) 270:13814-8. doi:10.1074/jbc.270.23.13814

31. Velazquez L, Mogensen KE, Barbieri G, Fellous M, Uzé G, Pellegrini S. Distinct domains of the protein tyrosine kinase tyk2 required for binding of interferon-alpha/beta and for signal transduction. JBiol Chem (1995) 270:3327-34. doi:10.1074/jbc.270.7.3327

32. Yan H, Piazza F, Krishnan K, Pine R, Krolewski JJ. Definition of the interferonalpha receptor-binding domain on the TYK2 kinase. J Biol Chem (1998) 273:4046-51. doi:10.1074/jbc.273.7.4046

33. Richter MF, Duménil G, Uzé G, Fellous M, Pellegrini S. Specific contribution of Tyk2 JH regions to the binding and the expression of the interferon alpha/beta receptor component IFNAR1. J Biol Chem (1998) 273:24723-9. doi:10.1074/jbc.273.38.24723

34. Chen M, Cheng A, Chen YQ, Hymel A, Hanson EP, Kimmel L, et al. The amino terminus of JAK3 is necessary and sufficient for binding to the common gamma chain and confers the ability to transmit interleukin 2-mediated signals. Proc Natl Acad Sci U S A (1997) 94:6910-5. doi:10.1073/pnas.94.13.6910
35. Lupardus PJ, Ultsch $M$, Wallweber H, Bir Kohli P, Johnson AR, Eigenbrot C. Structure of the pseudokinase-kinase domains from protein kinase TYK2 reveals a mechanism for Janus kinase (JAK) autoinhibition. Proc Natl Acad Sci U S A (2014) 111:8025-30. doi:10.1073/pnas.1401180111

36. Shan Y, Gnanasambandan K, Ungureanu D, Kim ET, Hammarén H, Yamashita K, et al. Molecular basis for pseudokinase-dependent autoinhibition of JAK2 tyrosine kinase. Nat Struct Mol Biol (2014) 21:579-84. doi:10.1038/ nsmb. 2849

37. Luo H, Rose P, Barber D, Hanratty WP, Lee S, Roberts TM, et al. Mutation in the Jak kinase JH2 domain hyperactivates Drosophila and mammalian Jak-Stat pathways. Mol Cell Biol (1997) 17:1562-71. doi:10.1128/MCB.17.3.1562

38. Saharinen P, Takaluoma K, Silvennoinen O. Regulation of the Jak2 tyrosine kinase by its pseudokinase domain. Mol Cell Biol (2000) 20:3387-95. doi:10.1128/MCB.20.10.3387-3395.2000

39. Casanova JL, Holland SM, Notarangelo LD. Inborn errors of human JAKs and STATs. Immunity (2012) 36:515-28. doi:10.1016/j.immuni.2012.03.016

40. Cacalano NA, Migone TS, Bazan F, Hanson EP, Chen M, Candotti F, et al. Autosomal SCID caused by a point mutation in the N-terminus of Jak3: mapping of the Jak3-receptor interaction domain. EMBO J (1999) 18:1549-58. doi:10.1093/emboj/18.6.1549

41. Schmalstieg FC, Leonard WJ, Noguchi M, Berg M, Rudloff HE, Denney RM, et al. Missense mutation in exon 7 of the common gamma chain gene causes a moderate form of X-linked combined immunodeficiency. J Clin Invest (1995) 95:1169-73. doi:10.1172/JCI117765

42. Russell SM, Johnston JA, Noguchi M, Kawamura M, Bacon CM, Friedmann M, et al. Interaction of IL-2R beta and gamma c chains with Jak1 and Jak3: implications for XSCID and XCID. Science (1994) 266:1042-5. doi:10.1126/science.7973658

43. Lupardus PJ, Skiniotis G, Rice AJ, Thomas C, Fischer S, Walz T, et al. Structural snapshots of full-length Jak1, a transmembrane gp130/IL-6/IL-6R $\alpha$ cytokine receptor complex, and the receptor-Jak1 holocomplex. Structure (2011) 19:45-55. doi:10.1016/j.str.2010.10.010

44. Wallweber HJA, Tam C, Franke Y, Starovasnik MA, Lupardus PJ. Structural basis of recognition of interferon- $\alpha$ receptor by tyrosine kinase 2 . Nat Struct Mol Biol (2014) 21:443-8. doi:10.1038/nsmb.2807

45. Ferrao R, Wallweber HJA, Ho H, Tam C, Franke Y, Quinn J, et al. The structural basis for class II cytokine receptor recognition by JAK1. Structure (2016) 24:897-905. doi:10.1016/j.str.2016.03.023

46. Zhang D, Wlodawer A, Lubkowski J. Crystal structure of a complex of the intracellular domain of interferon $\lambda$ receptor 1 (IFNLR1) and the FERM/SH2 domains of human JAK1. J Mol Biol (2016) 428(23):4651-68. doi:10.1016/j. jmb.2016.10.005

47. McNally R, Toms AV, Eck MJ. Crystal structure of the FERM-SH2 module of human Jak2. PLoS One (2016) 11:e0156218. doi:10.1371/journal.pone. 0156218

48. Ceccarelli DFJ, Song HK, Poy F, Schaller MD, Eck MJ. Crystal structure of the FERM domain of focal adhesion kinase. J Biol Chem (2006) 281:252-9. doi:10.1074/jbc.M509188200

49. Pearson MA, Reczek D, Bretscher A, Karplus PA. Structure of the ERM protein moesin reveals the FERM domain fold masked by an extended actin binding tail domain. Cell (2000) 101:259-70. doi:10.1016/S0092-8674(00)80836-3

50. Bradshaw JM, Waksman G. Molecular recognition by $\mathrm{SH} 2$ domains. $A d v$ Protein Chem (2002) 61:161-210. doi:10.1016/S0065-3233(02)61005-8

51. Ragimbeau J, Dondi E, Alcover A, Eid P, Uzé G, Pellegrini S. The tyrosine kinase Tyk2 controls IFNAR1 cell surface expression. EMBO J (2003) 22:537-47. doi:10.1093/emboj/cdg038

52. Radtke S, Haan S, Jörissen A, Hermanns HM, Diefenbach S, Smyczek T, et al. The Jak1 SH2 domain does not fulfill a classical SH2 function in Jak/STAT signaling but plays a structural role for receptor interaction and up-regulation of receptor surface expression. J Biol Chem (2005) 280:25760-8. doi:10.1074/ jbc.M500822200

53. McNally R, Eck MJ. JAK-cytokine receptor recognition, unboxed. Nat Struct Mol Biol (2014) 21:431-3. doi:10.1038/nsmb.2824

54. Haan S, Margue C, Engrand A, Rolvering C, Schmitz-Van de Leur H, Heinrich PC, et al. Dual role of the Jak1 FERM and kinase domains in cytokine receptor binding and in stimulation-dependent Jak activation. J Immunol (2008) 180:998-1007. doi:10.4049/jimmunol.180.2.998

55. Ashkenazy H, Abadi S, Martz E, Chay O, Mayrose I, Pupko T, et al. ConSurf 2016: an improved methodology to estimate and visualize evolutionary 
conservation in macromolecules. Nucleic Acids Res (2016) 44:W344-50. doi:10.1093/nar/gkw408

56. Dinerstein H, Lago F, Goujon L, Ferrag F, Esposito N, Finidori J, et al. The proline-rich region of the GH receptor is essential for JAK2 phosphorylation, activation of cell proliferation, and gene transcription. Mol Endocrinol (1995) 9:1701-7. doi:10.1210/mend.9.12.8614406

57. Avalos BR, Hunter MG, Parker JM, Ceselski SK, Druker BJ, Corey SJ, et al. Point mutations in the conserved box 1 region inactivate the human granulocyte colony-stimulating factor receptor for growth signal transduction and tyrosine phosphorylation of p75c-rel. Blood (1995) 85:3117-26.

58. Stahl N, Boulton TG, Farruggella T, Ip NY, Davis S, Witthuhn BA, et al. Association and activation of Jak-Tyk kinases by CNTF-LIF-OSM-IL-6 beta receptor components. Science (1994) 263:92-5. doi:10.1126/science.8272873

59. Kershaw NJ, Murphy JM, Liau NPD, Varghese LN, Laktyushin A, Whitlock EL, et al. SOCS3 binds specific receptor-JAK complexes to control cytokine signaling by direct kinase inhibition. Nat Struct Mol Biol (2013) 20:469-76. doi:10.1038/nsmb.2519

60. Brami-Cherrier K, Gervasi N, Arsenieva D, Walkiewicz K, Boutterin M-C, Ortega A, et al. FAK dimerization controls its kinase-dependent functions at focal adhesions. EMBO J (2014) 33:356-70. doi:10.1002/embj.201386399

61. Lietha D, Cai X, Ceccarelli DFJ, Li Y, Schaller MD, Eck MJ. Structural basis for the autoinhibition of focal adhesion kinase. Cell (2007) 129:1177-87. doi:10.1016/j.cell.2007.05.041

62. Watowich SS, Liu KD, Xie X, Lai SY, Mikami A, Longmore GD, et al. Oligomerization and scaffolding functions of the erythropoietin receptor cytoplasmic tail. J Biol Chem (1999) 274:5415-21. doi:10.1074/jbc.274.9.5415

63. Haan C, Hermanns HM, Heinrich PC, Behrmann I. A single amino acid substitution (Trp(666)->Ala) in the interbox 1/2 region of the interleukin-6 signal transducer gp130 abrogates binding of JAK1, and dominantly impairs signal transduction. Biochem J (2000) 349:261-6. doi:10.1042/0264-6021:3490261

64. Miura O, Cleveland JL, Ihle JN. Inactivation of erythropoietin receptor function by point mutations in a region having homology with other cytokine receptors. Mol Cell Biol (1993) 13:1788-95. doi:10.1128/MCB.13.3.1788

65. Haan C, Heinrich PC, Behrmann I. Structural requirements of the interleukin-6 signal transducer gp130 for its interaction with Janus kinase 1: the receptor is crucial for kinase activation. Biochem J (2002) 361:105-11. doi:10.1042/0264-6021:3610105

66. Constantinescu SN, Huang LJ-S, Nam H-S, Lodish HF. The erythropoietin receptor cytosolic juxtamembrane domain contains an essential, precisely
oriented,hydrophobicmotif.MolCell(2001)7:377-85.doi:10.1016/S1097-2765 (01)00185-X

67. Takai Y, Kitano K, Terawaki S-I, Maesaki R, Hakoshima T. Structural basis of the cytoplasmic tail of adhesion molecule CD43 and its binding to ERM proteins. J Mol Biol (2008) 381:634-44. doi:10.1016/j.jmb.2008.05.085

68. Hamada K, Shimizu T, Yonemura S, Tsukita S, Tsukita S, Hakoshima T. Structural basis of adhesion-molecule recognition by ERM proteins revealed by the crystal structure of the radixin-ICAM-2 complex. EMBO J (2003) 22:502-14. doi:10.1093/emboj/cdg039

69. Wei Z, Yan J, Lu Q, Pan L, Zhang M. Cargo recognition mechanism of myosin $\mathrm{X}$ revealed by the structure of its tail MyTH4-FERM tandem in complex with the DCC P3 domain. Proc Natl Acad Sci U S A (2011) 108:3572-7. doi:10.1073/ pnas. 1016567108

70. Li Y, Wei Z, Zhang J, Yang Z, Zhang M. Structural basis of the binding of merlin FERM domain to the E3 ubiquitin ligase substrate adaptor DCAF1. J Biol Chem (2014) 289:14674-81. doi:10.1074/jbc.M114.551184

71. Terawaki S-I, Maesaki R, Hakoshima T. Structural basis for NHERF recognition by ERM proteins. Structure (2006) 14:777-89. doi:10.1016/j.str.2006.01.015

72. Hamada K, Shimizu T, Matsui T, Tsukita S, Hakoshima T. Structural basis of the membrane-targeting and unmasking mechanisms of the radixin FERM domain. EMBO J (2000) 19:4449-62. doi:10.1093/emboj/19.17.4449

73. Wu L, Pan L, Wei Z, Zhang M. Structure of MyTH4-FERM domains in myosin VIIa tail bound to cargo. Science (2011) 331:757-60. doi:10.1126/ science.1198848

74. Li Y, Zhou H, Li F, Chan SW, Lin Z, Wei Z, et al. Angiomotin binding-induced activation of merlin/NF2 in the Hippo pathway. Cell Res (2015) 25:801-17. doi:10.1038/cr.2015.69

Conflict of Interest Statement: The authors are full-time employees of Genentech, Inc.

Copyright () 2017 Ferrao and Lupardus. This is an open-access article distributed under the terms of the Creative Commons Attribution License (CC BY). The use, distribution or reproduction in other forums is permitted, provided the original author(s) or licensor are credited and that the original publication in this journal is cited, in accordance with accepted academic practice. No use, distribution or reproduction is permitted which does not comply with these terms. 